Instability, Chaos and Predictability in Celestial Mechanics and Stellar Dynamics
Copyright 1992 by Nova Science Publishers, Inc. All rights of reproduction in any form reserved. ISBN 1-56072-054-9

\title{
APPLICATION OF SZEBEHELY'S INVERSE PROBLEM TO NON-STATIONARY DYNAMICAL SYSTEMS
}

\author{
G.T. Omarova and T.S. Kozhanov \\ Astrophysical Institute of the Kazakh Academy of Sciences, 480068, \\ Alma-Ata, Russia
}

\section{A BSTRACT}

A $f$ irst-order 1 inear partial differential equation is presented, giving the non-stationary potential functions $U=U$ $(x, y, t)$ which $g$ ive $r i s e$ to a given family of evoling planar or bits $f(x, y, t)=c$ in two-dimensional dynamical system. It is shown, that this equation is applied in celestial mechanics of variable mass.

\section{INTRODUCT ION}

A new approach to the classical inverse problem of finding the potential from the orbits wa made by $V$. Szehehely (1974).

Let

$$
f(x, y)=c=\text { const. }
$$

is a given monoparametric family of planar orbits in dynamical system determined by the equations of motion in rectangular coordinates $x$ and $y$

$$
\ddot{x}=u_{x}, \ddot{y}=u_{y}
$$

then the potential $U=U(x, y)$ may be determined from szebehely's equation 


$$
f_{x} U_{x}+f_{y^{\prime} y}+2 \frac{f_{x}^{2} f_{y y}-2 f x f_{x} f^{\prime}+f_{y}^{2} f x}{f_{x}^{2}+f_{y}^{2}}(U+h)=0
$$

where dots denote derivatives with respect to the time, subscripts correspond to partial derivatives and $h$ is the total energy per unit mass of the body.

In the present paper we shall consider the inver se problem for two-dimensional dissipative system.

\section{THE DER IVATION OF THE EQUATION}

Let us consider the two-dimensional dissipative system determined by the equations of motion

$$
\ddot{x}=U_{x}+\alpha \dot{x}, \ddot{y}=U_{y}+\alpha \dot{y},
$$

where $U=U(x, y, t)$ and $\alpha=\alpha(t)$ is an arbitrary function of time. These equations admit the non-stationary analogy of the angular momentum integral

$$
x \dot{y}-y \dot{x}=k, \quad k=\text { const. } \exp \left(\int_{t_{0}}^{t} \alpha(t) d t\right)
$$

For the family of orbits given by a twice differentiable function $f(x, y, t)=c=$ const., we have along each orbit

$$
\dot{x} f_{x}+\dot{y} f_{y}+f_{t}=0
$$

From equations (5) and (6) the components of the velocity may be expressed as

$$
\dot{x}=\frac{-k f y-x f t}{x f_{x}+y f_{y}},
$$

and

$$
\dot{y}=\frac{k f_{x}-y f_{t}}{x f_{x}+y f_{y}}
$$

The time-derivative of equation (6) is 


$$
\ddot{x f}_{x}+\ddot{y f}_{y}+\dot{x}^{2} f_{y y}+2 \dot{x} \dot{y} f_{x y}+2 \dot{x} f_{x t}+2 \dot{y f} y t+f_{t t}=0
$$

Substituting equations (4) and (7) into the equation (8) one obtains

$$
\begin{aligned}
& f_{x} U_{x}+f_{y} U_{y}+k \frac{f_{x}^{2} f y-2 f_{x y} f_{x}^{f} y+f_{y}^{2} f x}{\left(x f_{x}+y f_{y}\right)^{2}} \\
& +\frac{2 k f_{t}\left(f_{y} f_{x x}\right.}{\left.-f_{x} f_{x y}\right) x+\left(f_{y} f_{x y}-f_{x} f_{y y}\right) y} \\
& +2 k \frac{f_{x} f y t-f y}{x f_{x}+y f_{y}}+f_{t}^{2} \frac{x^{2} f_{x x}+2 x y f_{x y}+y^{2} f_{y y}}{\left(x f_{x}+y f_{y}\right)^{2}} \\
& -2 f_{t} \frac{x f_{x t}+y f_{y t}}{x f_{x}+y f_{y}}-\alpha f_{t}+f_{t t}=0
\end{aligned}
$$

Equation (9) in polar coordinates $r$ and $\theta$ is

$$
\begin{aligned}
f_{r} U_{r} & +\frac{f_{\theta}}{r^{2}} U_{\theta}+\frac{k^{2}}{r^{5} f_{r}^{2}}\left(r f_{r r} f_{\theta}^{2}+r f_{r}^{2} f_{\theta \theta}\right. \\
& \left.-2 r f_{r} f_{r \theta} f_{\theta}+r^{2} f_{r}^{3}+2 f_{r} f_{\theta}^{2}\right)+2 \frac{k f_{t}}{r^{3} f_{r}^{2}}\left(f_{r} f_{\theta}\right. \\
& \left.-r f_{r} f_{r \theta}+r f_{r r} f_{\theta}\right)-\frac{2 f_{r t} f_{t}}{r}-\alpha f_{t}+f_{t t}=0
\end{aligned}
$$

The equation (9) (or (10)) is a first-order' Inbar partial differential equation, giving the non-stationary potentials. The solution of this equation is not unique.

\section{AN EXAMPLE}

As an example of solution of the equation (lo) one may consider the motion along evolving spiral orbits

$$
f(r, \theta, t)=r \gamma(1+e \cos \theta)=P=\text { const., }
$$

where $\gamma=\gamma(t)$ is a given function of time, e = const. 
Substituting (11) in (10) we obtain

$$
(1+e \cos \theta) u_{r}-\frac{\gamma e}{r} \sin \theta u_{\theta}+\frac{k^{2}}{r^{3}}-W P=0,
$$

where

$$
\dot{w}=w(t)=\frac{\alpha \dot{\gamma}}{\gamma}+2 \frac{\dot{\gamma}^{2}}{\gamma^{2}}-\frac{\ddot{\gamma}}{\gamma}
$$

Equation (12) can be solved directly by the method of characteristic s.

Then

$$
\frac{d r}{\gamma(1+e \cos \theta)}=-\frac{r d \theta}{\gamma e \sin \theta}=\frac{d U}{-\frac{k^{2} \gamma}{r^{3}}+\text { r.P }}
$$

The general solution of equation (14) is

$$
U=\frac{k^{2} r}{p \cdot r}+\frac{w \cdot r^{2}}{2}+\phi\left(\frac{r^{e}(1-\cos \theta)}{(\sin \theta)^{1-e}}\right)
$$

where $\phi$ is an arbitrary function of its argument.

THE EQUATIONS OF MODEL PROBLEMS IN CELESTIAL MECHANICS OF VAR IARLE MASS

Let us put in (15) $\phi=0$, then equation (15) determines the force of the form

$$
\vec{F}=-\frac{k^{2} \gamma}{\operatorname{Pr}^{3}} \vec{r}+w \vec{r}
$$

From equation (16) under various values of the ifunctions $k(t), \alpha(t), \gamma(t)$ we can obtain the following equations of motion:

1. When $k(t)=\sqrt{G M(t)}, \gamma(t)=P=$ const.,

then we have from $(16)$ the equation of aperiodic motion along a conic section

$$
\frac{d^{2} \vec{r}}{d t^{2}}=-G M(t) \frac{\vec{r}}{r^{3}}+\frac{1}{2 M} \frac{d M}{d t} \frac{d \vec{r}}{d t} ;
$$


2. When $\alpha(t)=0, k(t)=\sqrt{P}=$ const., $\gamma(t)=\mu(t)$, then in this case we obtain the equation of the model problem for a spiral motion

$$
\frac{d^{2} \vec{r}}{d t^{2}}=-\frac{\mu \vec{r}}{r^{3}}+\mu \vec{r} \frac{d^{2}}{d t^{2}}\left(\frac{1}{\mu}\right) ;
$$

3. When

$$
k(t)=\sqrt{\frac{\mu(t)}{\gamma(t)}}, \quad \gamma(t)=\frac{1}{\gamma(t)} .
$$

then we receive the equation of motion of the model problem of the evolution of binary system inside gravitating resistant medium

$$
\frac{d^{2} \vec{r}}{d t^{2}}=-\mu \frac{\vec{r}}{r^{3}}+\frac{1}{2}\left(\frac{\dot{\mu}}{\mu}+\frac{\dot{\gamma}}{\gamma}\right) \vec{r}+\left[\frac{\ddot{\gamma}}{\gamma}-\frac{1}{2}\left(\frac{\dot{\mu}}{\mu}+\frac{\dot{\gamma}}{\gamma}\right) \frac{\dot{\gamma}}{\gamma}\right] \vec{r}
$$

The equations (18), (20), (22) play important role in celestial mechanics of variable mass.

\section{REFERENCES}

1 Szebehely, V., (1974), 'On the Determination of Potential', in Proc. Int. Mtg. on the Rotation of the Earth, E.Prover bio (Ed.), Bologna.

2 Omarov, T.B. and Minglibaev, M. (1983), in Dynamical Trapping and Evolution in the Solar system, IAU Collog. N.74, Saloniki. 